\title{
ARTICLE
}

\section{The characteristics of traumatic spinal cord injuries at a referral hospital in Northern Tanzania}

\author{
Sakina Mehboob Rashid ${ }^{1}$, Mubashir Alavi Jusabani ${ }^{2}$, Faiton Ndesanjo Mandari ${ }^{3}$ and Marieke Cornelia Johanna Dekker ${ }^{4,5}$
}

STUDY DESIGN: Retrospective study.

OBJECTIVES: To describe the epidemiology of traumatic spinal cord injury (TSCI) in Northern Tanzania.

SETTING: Main referral hospital for Northern Tanzania.

METHODS: A retrospective review of $125 \mathrm{TSCl}$ cases admitted to Kilimanjaro Christian Medical Centre (KCMC) over a period of 5 years.

RESULTS: We included 125 patients in the sample size, with a male majority of 107 (85.6\%), giving a male-to-female ratio of 5.9:1. The mean age at injury was $39.9( \pm 16.0)$ years. Overall, $66(52.8 \%)$ were farmers and an overwhelming majority $(109(87.2 \%))$ were referrals from peripheral medical centres. Fall injuries accounted for 65 (52.0\%) of the admissions and 49 (39.2\%) were injured due to a Road Traffic Accident (RTA). Severity of injury was classified according to the American Spinal Injury Association Impairment Scale (AIS) and 57 (45.6\%) injuries were categorised as AIS A and $68(54.4 \%)$ as AIS BCD. Cervical level injuries (59 (47.2\%)) were the most frequent among the cohort. The in-hospital mortality rate was $24.1 \%$.

CONCLUSION: The majority of patients affected by TSCls were young males and the most common cause was fall injuries. Cervical level injuries were the most common and in-hospital mortality was high.

Spinal Cord Series and Cases (2017) 3, 17021; doi:10.1038/scsandc.2017.21; published online 11 May 2017

\section{INTRODUCTION}

The first mention of spinal cord injuries (SCls) can be traced back to 1600 years Before the Common Era (BCE), appearing in a medical text recovered from Ancient Egypt, which labelled the condition as an 'ailment not to be treated'. 'More than three millennia later, this condition remains formidable in its impact on the lives of those afflicted even though treatment options have evolved beyond the historical 'hands off' approach with the assumption that nothing more could be done. SCls encompass a wide range of conditions, not all of which are permanently disabling. Conditions representing one end of the spectrum include cervical cord neuropraxia which manifests with temporary symptoms, ${ }^{2}$ while permanent disruption of spinal cord function due to injury presents a clinical picture of irreversible neurological disability below the level of the lesion. Of note is the SCIWORA (Spinal Cord Injury Without Radiographic Abnormality) phenomenon; the acronym refers to the clinical presentation of traumatic myelopathy without evidence of correlating pathology on X-ray or CT-Scan. The condition is most prevalent in the paediatric population below 8 years of age and accounts for $6-19 \%$ of injuries in children. ${ }^{3}$

$\mathrm{TSCl}$ annual incidence rates demonstrate great variability across published data for different regions; figures as high as 53 cases per million were put forward by a Canadian paper while a Dutch epidemiological study presented a much lower statistic of 13 cases per million. ${ }^{4,5}$ At present, data for the global prevalence and incidence of traumatic spinal cord injuries (TSCls) are incomplete since across the world, various large scale studies aiming to provide accurate estimates were not entirely succesful. ${ }^{6}$
Of the numerous challenges faced by investigators, of note are; the lack of a standardised process of assessing $\mathrm{SCls}$ across different regions in the world. ${ }^{6}$ In addition, the complexities of data extrapolation from small-scale studies to present statistics for national incidence and prevalence rates and the predominance of studies being conducted in the world's more developed regions also add to the challenge. ${ }^{6}$ The likelihood of underestimation also remains, since $\mathrm{TSCls}$ are associated with a high mortality before patients are admitted for diagnosis and care which will lead to underreporting in those areas of the world where ambulances or other on-the-spot emergency care is suboptimal or not there at all. ${ }^{6}$

There is a paucity of epidemiological data illustrating the burden of TSCls within Africa as illustrated in Draulans et al.'s ${ }^{7}$ work, which is especially true for the East African countries. Most publications have reported results from studies based in Nigeria and South Africa. ${ }^{7}$ This study aimed to present a comprehensive perspective on the burden of TSCls received by the academic referral hospital KCMC in the Eastern African country of Tanzania as well as an attempt to contribute to the national and global map detailing $\mathrm{TSCl}$ epidemiology.

\section{METHODS}

Setting and study population

The study was based in the academic referral hospital of KCMC, located in Moshi, Kilimanjaro Region, Tanzania. KCMC is one of four consultant level hospitals in the country and the main referral centre for the North-East of Tanzania. Tanzania is one of the low

\footnotetext{
${ }^{1}$ Kilimanjaro Christian Medical University College, Moshi, Tanzania; ${ }^{2}$ Kilimanjaro Christian Medical Centre, Moshi, Tanzania; ${ }^{3}$ Department of Orthopedics and Traumatology, Kilimanjaro Christian Medical Centre, Moshi, Tanzania; ${ }^{4}$ Department of Internal Medicine, Department of Pediatrics and Child Health, Kilimanjaro Christian Medical Centre, Moshi, Tanzania and ${ }^{5}$ Department of Neurology, Radboudumc Nijmegen, Nijmegen, The Netherlands. 
income countries ${ }^{8}$ ranking 186th in terms of its gross national income per capita ${ }^{9}$ as of 2015 and has a population of 53.5 million inhabitants. ${ }^{9}$

The medical facilities at KCMC are a reflection of a limited health budget for the country. There is no magnetic resonance imaging scanner in the hospital and no on-call neurosurgical expertise-a situation which applies to the entire country except the Eastern coastal city of Dar es Salaam.

The study period ranged from January 2011 to December 2015.

All TSCl patients admitted to the KCMC Orthopedics Department during the study duration were included in the sample size. Because the institution based nature of this study and the location in one of the world's low income countries, ${ }^{8}$ the results are unlikely to be an accurate representation of TSCls in Northern Tanzania as a significant number of patients may be admitted to different medical centres, and a number will have succumbed to injuries before admission due to the virtual absence of on-site emergency medical care.

\section{Methodology and variables}

This was a retrospective hospital-based study. The Medical Records Department Registers were utilised to compile a list of relevant patient file numbers which were then retrieved from the Medical Record's Archive. Each patient file was methodically scrutinised to extract data concerning the patient sociodemographic characteristics as well as aetiology, level and severity of injury. Following file retrieval, the expected sample size $(n=250)$ was significantly reduced as a number of files were misplaced, patients had to be excluded because of missing data and some patients had been misdiagnosed.

Hospital protocol dictates that patients with severe head injuries are admitted to the General Surgery Wards and not Orthopedics, which implies a potential for overlooking the spinal injuries in these patients. For this reason, the number of $\mathrm{TSCl}$ would have been underestimated in the final sample size.

An internally developed TSCI Management Protocol is in place at KCMC which requires every $\mathrm{TSCl}$ patient's neuromuscular assessment and neurological level to be documented upon admission and during every week thereafter. Patients are then characterised as having complete or incomplete $\mathrm{TSCls}$ based on the American Spinal Injury Association Impairment Scale (AIS) categories. Therefore, for the purpose of this study, severity was classified as either AIS A, AIS BCD or AIS E.

The neurological level of injury was categorised as either cervical, thoracic or lumbar.

\section{Data analysis}

Data were entered into Statistical Package for the Social Sciences version 23 software. All variables were eventually analysed descriptively, because of the limited sample size, continuous variables have been presented in the form of means ( \pm s.d.) and categorical variables have been presented as frequencies.

\begin{tabular}{|c|c|c|}
\hline Age Group (Years) & Number & Percent $(\%$ \\
\hline $1-15$ & 5 & 4.0 \\
\hline $16-30$ & 35 & 28.0 \\
\hline $33-45$ & 41 & 32.8 \\
\hline $46-60$ & 28 & 22.4 \\
\hline $61-75$ & 14 & 11.2 \\
\hline $76-90$ & 2 & 1.6 \\
\hline
\end{tabular}

Ethics

This research study was approved of by the Medical Ethics Committee of Kilimanjaro Christian Medical Centre, Moshi, United Republic of Tanzania.

\section{RESULTS}

Study population

A total of 125 patients were included in our study sample, with a male majority of 107 (85.6\%), giving a male-to-female ratio of 5.9:1.

The mean age at injury was $39.9( \pm 16.0)$ years, with a range of 5-77 years. The most frequently affected age group was 31-45 years (41 (32.8\%)) (Table 1). Overall, 66 patients (52.8\%) were farmers and an overwhelming majority (109 (87.2\%)) were referrals from peripheral medical centres. Fall injuries accounted for $65(52.0 \%)$ of the admissions and 49 (39.2\%) were injured due to a RTA (Table 2).

\section{Injury characteristics}

Severity of injury was classified according to the AIS classification and 57 (45.6\%) injuries were categorised as AIS A and 68 (54.4\%) as AIS BCD (Table 3). Cervical level injuries (59 (47.2\%)) were the most frequent among the cohort. Thoracic level injury was diagnosed in 28 (22.4\%) individuals and in 38 (30.4\%), the injury was localised to the lumbar level. Over the 5-year study period, only $2(1.6 \%)$ patients out of 125 underwent surgical decompression of the spinal cord. The mean duration of stay in the hospital was $71.6( \pm 76.2)$ days with a median duration of stay 46 days long. The short-term mortality rate before discharge was $24.1 \%$ (Tables 4 and 5).

\section{DISCUSSION}

To the best of our knowledge based on online medical literature databases, this is the first report from Tanzania detailing the characteristics of TSCls. To highlight the burden of this devastating injury is important as it mostly concerns young family breadwinners who form the backbone of an economically fragile society.

The male preponderance noted in the study sample is in keeping with numerous publications which examined $\mathrm{TSCl}$

Table 2. Causes of TSCl

\begin{tabular}{lcc}
\hline Cause of Injury & Number & Percentage \\
\hline Fall Injuries & 65 & 52.0 \\
RTAs & 49 & 39.2 \\
Other & 11 & 8.8 \\
\hline
\end{tabular}

Abbreviation: RTA, Road Traffic Accidents. Other: Violence Related Incidents (3), Mining Accidents (2), Weight Fell on Head (3), Car Jack Failure (1), Cart Running Over Neck (1), Milling Machine Accident (1).

Table 3. Neurological Level of Injury and Injury Severity

\begin{tabular}{lccr}
\hline & \multicolumn{2}{c}{ Injury Severity } & Total \\
\cline { 2 - 3 } & AIS A & AIS BCD & \\
\hline Level of Injury (Neurological) & & \\
Cervical & 28 & 31 & 59 \\
Thoracic & 23 & 15 & 38 \\
Lumbar & 6 & 22 & 28 \\
Total & 57 & 68 & 125 \\
\hline Abbreviation: AIS, American Spinal Injury Association Impairment Scale.
\end{tabular}




\begin{tabular}{|c|c|c|c|}
\hline & \multicolumn{2}{|c|}{ Mortality Before Discharge } & \multirow[b]{2}{*}{ Total } \\
\hline & Alive & Deceased & \\
\hline \multicolumn{4}{|c|}{ Level of Injury (Neurological) } \\
\hline Cervical & 36 & 23 & 59 \\
\hline Thoracic & 30 & 8 & 38 \\
\hline Lumbar & 27 & 1 & 28 \\
\hline Total & 93 & 32 & 125 \\
\hline
\end{tabular}

Table 5. Injury Severity and Mortality Before Discharge

\begin{tabular}{|c|c|c|c|}
\hline & \multicolumn{2}{|c|}{ Mortality Before Discharge } & \multirow[t]{2}{*}{ Total } \\
\hline & Alive & Deceased & \\
\hline \multicolumn{4}{|c|}{ Injury Severity } \\
\hline AIS A & 29 & 28 & 57 \\
\hline AIS BCD & 64 & 4 & 68 \\
\hline Total & 93 & 32 & 125 \\
\hline
\end{tabular}

epidemiology. ${ }^{6,10}$ Male-to-female ratios of up to 13.5:1 have been reported by a study based in rural India, however the trend of consistently higher incidences recorded for males in TSCl studies only emerges beyond the paediatric age group. ${ }^{10,11}$ This is a reflection of ubiquitous gender roles and a higher exposure to risk factors which become progressively significant with into adulthood.

Although $52.8 \%$ of the cohort had reported that they are farmers by profession, it should be noted that it is often a blanket term used locally to denote either unemployment in retired individuals or a lifestyle of subsistence farming to sustain the food consumption of a family. Furthermore, the income of such individuals would be either semi-annual or annual and heavily dependent on crop yield and seasonal weather patterns.

The typical profile is thus that of a previously able man, the breadwinner of the family who has to face irreversible destruction of quality of life both as an individual and of his immediate dependants.

An overwhelming majority (87.2\%) of patients were referrals from peripheral health centres. This is a significant observation in our setting as it often implies unsteady transfer positions for long distances, on unpaved roads while accompanied by health personnel who are not trained to handle patients with unstable spinal injuries.

A publication dating back to 1985 which was based in KCMC, retrospectively examined 47 patients who were diagnosed with traumatic paraplegia. ${ }^{12}$ The two main causes of injury had been recorded as fall injuries (40.4\%) followed by RTAs $(23.4 \%){ }^{12}$ It is rather striking that despite a tremendous increase in road traffic levels and reports of RTAs in Moshi region over the past few decades, the leading aetiologies of injury for a similar-albeit more widely inclusive-cohort gathered from the same institution have remained unchanged-fall injuries (52.0\%) and RTAs (39.2\%).

A significant percentage of injuries in developed regions of the world result from RTAs, in contrast to fall injuries which comprise the majority of aetiological classifications in the developing countries. ${ }^{13}$ The circumstances which result in injury are generally a representation of the culture, lifestyle and environment of the patient and we believe this factor has contributed to the aetiology of injury more than the increased road traffic congestion in Moshi region over the past few decades.
Table 6. Mortality Rates Across Different Countries

\begin{tabular}{|c|c|c|c|}
\hline Country & $\begin{array}{l}\text { Mortality } \\
\text { rate (\%) }\end{array}$ & Setting & $\begin{array}{l}\text { Time } \\
\text { Period }\end{array}$ \\
\hline South Africa ${ }^{17}$ & 2.2 & Specialised SCI Unit & 11 Years \\
\hline Canada $^{18}$ & 10.7 & SCI Registry & 10 Years \\
\hline Australia $^{18}$ & 14.3 & SCI Registry & 10 Years \\
\hline $\begin{array}{l}\text { United States } \\
\text { of America }{ }^{18}\end{array}$ & 16.2 & SCI Registry & 10 Years \\
\hline Nigeria $^{16}$ & 17.5 & Hospital Based & 15 Years \\
\hline Botswana ${ }^{22}$ & 20.0 & Hospital Based & 2 Years \\
\hline
\end{tabular}

AIS class A injuries accounted for $45.6 \%$ of the study cohort. The bleak outlook for patients who have sustained a complete $\mathrm{TSCl}$ is not only limited to the poorer functional outcomes. Clinical progress analysis of TSCl patients in a Nigerian study demonstrated a higher rate of complications recorded for patients who have suffered a complete TSCl verses an incomplete injury. ${ }^{14}$

Cervical level injuries (47.2\%) were the most common in the study cohort-a finding which was consistent with multiple publications which classified TSCls according to the segment of the cord injured; Denmark (51.0\%), Estonia (59.4\%), Iceland (57.0\%), Ireland (50.0\%), Norway (53.0\%), Romania (57.7\%), Turkey (31.7\%), Japan (74.3\%), Australia (57.7\%) and New Zealand (52.3\%). ${ }^{6}$

The loss of any degree of independence is a distressing experience for a once able and fully functional adult, though it is the level of injury which remains amongst the primary determinants of the degree of disability. Patients with high tetraplegia $(\mathrm{C} 1-\mathrm{C} 4)$ if surviving the high chance of respiratory insufficiency which has no other treatment options in our setting, like chronic or home ventilation, will remain dependent for the activities of daily living. ${ }^{15}$ On the other hand, individuals with incomplete injuries in the C5-T1 region may be able to accomplish a variable number of these activities with complete independence or assistive technology depending on the exact level of the lesion. ${ }^{15}$

$\mathrm{KCMC}$ is home to the only spinal cord injury rehabilitation unit in Tanzania. At present, due to the highest potential for regaining limited independence in activities of daily living, only patients with thoraco-lumbar region spinal cord injuries are enroled in the unit. This often means that cervical level $\mathrm{TSCl}$ patients will be nursed by a family member following discharge from the wards. Frequently, this complex care is suboptimal.

The mortality rate for this cohort was slightly higher then that put forward by a study based in Lagos (17.5\%), but significantly greater than a publication from Cape Town (2.2\%). ${ }^{16,17}$ The South African study collected data from patients admitted to a dedicated $\mathrm{SCl}$ unit and the likely explanation for the dissimilar statistics may well be the admission criteria to the unit which considered injury severity, requirement of surgical intervention and likely outcome following a plateau of neurological recovery. ${ }^{17}$

The bleak prognosis of a severe $\mathrm{TSCl}$ is compounded by the lack of diagnostic and interventional facilities at KCMC. Without an magnetic resonance imaging scan and no neurosurgical expertise at the centre, most patients are managed conservatively. Over the 5 -year study period, only 2 (1.6\%) patients out of 125 underwent surgical decompression of the spinal cord and the short term mortality rate before discharge in the study cohort was $24.1 \%$.

Neurosurgical services are limited at KCMC, except for those conditions which can be managed by General Surgeons such as subdural haematomas, abscess drainage or ventriculo-peritoneal shunting. Also, spinal stabilisation surgery is often not conducted by orthopaedic surgeons due to lack of necessary equipment. Referral to specialised centres is further hampered by cost of travel, hospital stay and expert treatment, leaving this beyond reach of a vast majority of patients. 
High-income countries report 10-year mortality rates from $\mathrm{SCl}$ registries of $16.2 \%$ (USA), $14.3 \%$ (Australia) and $10.7 \%$ (Canada); figures which are in harsh contrast to trends noted in SSA countries where a majority of fatalities following TSCls are reported within 1 year $^{18}$ (Table 6). A Nigerian study reported a 1 -year mortality rate of $34.3 \%$ and a publication based in Zimbabwe put forward a much higher figure of up to $49 \%{ }^{19,20}$

The saddening statistics of this study have demonstrated the importance of primary prevention; the risks of fall injuries need to be stressed via public education and road safety rules require strict implementation.

A major limitation of the study was the retrieval of the patient's records. The file archiving system at KCMC is manually arranged, with a limited number of personnel working in File Retrieval. Coupled with an annual patient turnover upwards of 150000 , a significant number of files were misplaced and thus the patient data could not be included in the study sample. ${ }^{21}$ Missing data for the above reasons in unfortunately not an uncommon problem in resource limited settings.

An important problem that was also encountered during the retrospective review was the recurrence of misdiagnosis which meant that the initial sample size had been factitiously high. Although the number missing was large, we do not expect a bias due to differential misclassification of files related to a certain injury severity or level.

To conclude, TSCls represent a major burden to the health sector in Northern Tanzania and close to one in every four patients will succumb to their injuries. Although there are no local statistics detailing the disability adjusted life years (DALYs) and years lived with disability (YLDs) for TSCl patients, the population in Moshi consists largely of a rural community with the majority of the citizens relying on subsistence farming for their income. The demographic profile of a typical patient is strikingly similar to that of an average working male, thus the financial burden of this devastating injury is not only limited to the health budget, but the DALYs and YLDs also ripple across the local economy in terms of loss of financially productive years.

A national initiative to quantify the burden of TSCls in the form of a registry would serve multiple benefits. Apart from providing a comprehensive illustration of the national burden of the condition and the resources required to facilitate the essential multidisciplinary management, prevalence data will facilitate follow-up of individuals who are living with neurological deficits following TSCls. This will ease the provision of psychological support as well as occupational and physical therapy to enable patients to come to terms with their circumstances.

Suffering a TSCl is a devastating experience for an individual and as it is a condition unrestrained by social or geographical margins, it remains a significant threat to public health. For the patient, it often signifies the end of independent living and for a clinician, a manifestation of the disrupted intricacies of the spinal cord. The age-old aphorism-prevention is better than cure-certainly rings true.

\section{COMPETING INTERESTS}

The authors declare no conflict of interest.

\section{REFERENCES}

1 Schiller M, Mobbs R. The historical evolution of the management of spinal cord injury. J Clin Neurosci 2012; 19: 1348-1353.

2 Torg JS, Pavlov HE, Genuario SE, Sennett B, Wisneski RJ, Robie BH et al. Neurapraxia of the cervical spinal cord with transient quadriplegia. J Bone Joint Surg Am 1986; 68: 1354-1370.

3 Szwedowski D, Walecki J. Spinal cord injury without radiographic abnormality (SCIWORA)-clinical and radiological aspects. Pol J Radiol 2014; 79: 461-464.

4 Noonan VK, Fingas M, Farry A, Baxter D, Singh A, Fehlings MG et al. Incidence and prevalence of spinal cord injury in Canada: a national perspective. Neuroepidemiology 2012; 38: 219-226.

5 Van Asbeck FW, Post MW, Pangalila RF. An epidemiological description of spinal cord injuries in The Netherlands in 1994. Spinal cord 2000; 38: 420-424.

6 Singh A, Tetreault L, Kalsi-Ryan S, Nouri A, Fehlings M. Global prevalence and incidence of traumatic spinal cord injury. Clin Epidemiol 2014; 6: 309-331.

7 Draulans N, Kiekens C, Roels E, Peers K. Etiology of spinal cord injuries in Sub-Saharan Africa. Spinal Cord 2011; 49: 1148-1154.

8 Tanzania|Data [Internet]. Data.worldbank.org. 2016 [cited 18 December 2016]. Available from http://data.worldbank.org/country/tanzania.

9 Gross national income per capita 2015, Atlas method and PPP [Internet]. The World Bank. 2016 [cited 18 December 2016]. Available from http://databank. worldbank.org/data/download/GNIPC.pdf.

10 Ning GZ, Wu Q, Li YL, Feng SQ. Epidemiology of traumatic spinal cord injury in Asia: a systematic review. J Spinal Cord Med 2012; 35: 229-239.

11 Vogel LC, Betz RR, Mulcahey MJ. Spinal cord injuries in children and adolescents. Handbook of Clinical Neurology 2012; 109: 131-148.

12 Fisk N, Hulme-Moir I, Scrimgeour EM, Schlabach WE. Traumatic paraplegia in northern Tanzania. Trop Doct 1985; 15: 23-26.

13 Chiu WT, Lin HC, Lam C, Chu SF, Chiang YH, Tsai SH. Review paper: epidemiology of traumatic spinal cord injury: comparisons between developed and developing countries. Asia Pac J Public Health 2010; 22: 9-18.

14 Kawu AA, Alimi FM, Gbadegesin AAS, Salami AO, Olawepo A, Adebule TG et al. Complications and causes of death in spinal cord injury patients in Nigeria. West Afr J Med 2011; 30: 301-304.

15 WORLD HEALTH ORGANISATION. International Perspectives on Spinal Cord Injury. WHO Press: Geneva, Switzerland, 2013.

16 Obalum DC, Giwa SO, Adekoya-Cole TO, Enweluzo GO. Profile of spinal injuries in Lagos, Nigeria. Spinal Cord 2009; 47: 134-137.

17 Sothmann J, Stander J, Kruger N, Dunn R. Epidemiology of acute spinal cord injuries in the Groote Schuur Hospital Acute Spinal Cord Injury (GSH ASCI) Unit, Cape Town, South Africa, over the past 11 years. S Afr Med J 2015; 105: 835-839.

18 Cripps RA, Lee BB, Wing P, Weerts E, Mackay J, Brown D. A global map for traumatic spinal cord injury epidemiology: towards a living data repository for injury prevention. Spinal Cord 2011; 49: 493-501.

19 Nwadinigwe CU, lloabuchi TC, Nwabude IA. Traumatic spinal cord injuries (SCI): a study of 104 cases. Niger J Med 2004; 13: 161-165.

20 Levy LF, Makarawo S, Madzivire D, Bhebhe E, Verbeek N, Parry O. Problems, struggles and some success with spinal cord injury in Zimbabwe. Spinal Cord 1998; 36: 213-218.

21 TANZANIA, KILIMANJARO CHRISTIAN MEDICAL CENTRE(2015) Kilimanjaro Christian Medical Centre Annual Report 2014. Kilimanjaro, Tanzania. [Cited: 2nd February, 2017]. Available on http://www.kcmc.ac.tz/downloads/annual_report_ 2014.pdf.

22 Löfvenmark I, Norrbrink C, Nilsson-Wikmar L, Hultling C, Chakandinakira S, Hasselberg M. Traumatic spinal cord injury in Botswana: characteristics, aetiology and mortality. Spinal cord 2015; 53: 150-154. 